\title{
Utilité d'une évaluation infirmière systématique du risque de neutropénie fébrile induite par la chimiothérapie
}

par Kelley Moore, inf., et Barry Fortner, Ph.D.

\section{Abrégé}

Les lignes directrices fondées sur les preuves recommandent que les patients à risque élevé ( $\geq 20 \%$ ) de neutropénie fébrile (NF) reçoivent des facteurs de croissance hématopoïétiques à titre prophylactique (Aapro et al., 2006; Koroukis et al., 2008; National Comprehensive Cancer Network [NCCN], 2008; Smith et al., 2006). Nous avons étudié l'utilité de faire effectuer par les infirmières une évaluation systématique du risque de NF chez les nouveaux patients avant le commencement de la chimiothérapie. Quinze infirmières ont utilisé un outil normalisé afin d'évaluer le risque de NF chez 150 patients. Chez $94 \%$ des patients ainsi examinés, les infirmières ont décelé des facteurs de risque exigeant des interventions en vue de réduire l'incidence de la NF. Lors de la phase finale d'évaluation de l'outil, $67 \%$ des infirmières ont déclaré que l'emploi d'un outil normalisé les avait aidées à identifier les patients à risque de NF, et $73 \%$ d'entre elles projetaient d'évaluer systématiquement le risque de NF. Les infirmières trouveront que l'évaluation du risque de NF au moyen d'une liste de contrôle normalisée avant le commencement de la chimiothérapie est une procédure à la fois réalisable et fort utile.

La neutropénie induite par la chimiothérapie et ses conséquences sont une préoccupation constante des infirmières en oncologie. Selon les estimations, la neutropénie fébrile (NF) touche au moins de 25-40 \% des patients vierges de tout traitement recevant des protocoles de chimiothérapie courants (Dale, 2002). Environ un tiers des patients atteints de NF développent une infection confirmée par analyse microbiologique (Cordonnier et al., 2005; Gáytan-Martinez et al., 2005) et, au Canada, on dénombre, chaque année, approximativement 7000 hospitalisations pour cause de neutropénie (Desjardins \& Meilleur, 2005). Bien que la majorité des patients exigeant une hospitalisation ont des résultats favorables, la mortalité due aux hospitalisations liées à la NF s'élève à 7-10 \% (Caggiano, Weiss, Rickert \& Linde-Zworble, 2005; Kuderer, Dale, Crawford, Cosler \& Lyman, 2006). La neutropénie fébrile entraîne souvent des réductions des doses et/ou des retards dans l'administration des doses durant le traitement du cancer du sein de stade précoce

\section{Utility of routine nurse assessment of the risk of chemotherapy-induced febrile neutropenia}

\section{Abstract}

Evidence-based guidelines recommend that patients at high risk $(\geq 20 \%)$ for febrile neutropenia (FN) should receive prophylactic colony-stimulating factors (Aapro et al., 2006; Kouroukis et al., 2008; National Comprehensive Cancer Network [NCCN], 2008; Smith et al., 2006). We studied the utility of having nurses routinely assess FN risk in new patients before the initiation of chemotherapy. Fifteen nurses used a standardized tool to evaluate FN risk in 150 patients. In 94\% of patients studied, nurses detected risk factors that prompted interventions to reduce the incidence of FN. On final evaluation, 67\% of nurses said the use of a standardized tool helped them to identify patients at risk for FN, and $73 \%$ planned to assess FN risk routinely. Thus, it is feasible and valuable for nurses to assess FN risk using a standardized checklist prior to the initiation of chemotherapy.
(Lyman, Dale \& Crawford, 2003) ou du lymphome non hodgkinien (Lyman, Dale, Friedberg, Crawford \& Fisher, 2004). Des preuves toujours plus nombreuses indiquent que la modification des doses diminue la possibilité de traiter la maladie à long terme et raccourcit la survie des patients porteurs de tumeurs potentiellement guérissables (Bonadonna, Valagussa, Moliterni, Zambetti \& Brambilla, 1995; Budman et al., 1998; Epelbaum et al., 1990; Kwak, Halpern, Olshen \& Horning, 1990; Lyman, 2005).

Un essai comparatif aléatoire de phase III a démontré qu'une prophylaxie primaire au moyen d'un facteur de stimulation des colonies granulocytaires (G-CSF) réduit de manière significative l'incidence de la NF ainsi que les hospitalisations dues à cette dernière et l'utilisation d'antibiotiques intraveineux lorsqu'elle est donnée en même temps qu'un protocole de chimiothérapie modérément myélosuppresseur (Vogel et al., 2005). À la lumière de ces résultats, trois ensembles de lignes directrices sur l'utilisation des CSF ont été publiés ou actualisés par diverses organisations américaines et internationales (Aapro et al., 2006; National Comprehensive Cancer Network [NCCN], 2008; Smith et al., 2006). En outre, des recommandations ont également été publiées au Canada concernant l'utilisation de soins de soutien dans la prise en charge de la neutropénie (Koroukis et al., 2008). Selon ces lignes directrices, le consensus international est qu'il convient d'évaluer le risque de NF avant même de démarrer le premier cycle de chimiothérapie. De plus, ces lignes directrices stipulent qu'un CSF prophylactique doit être également donné si le protocole de chimiothérapie est associé à un risque de $\mathrm{NF} \geq 20 \%$ ou si le risque de NF due à la chimiothérapie se situe entre 10 et $20 \%$, et en présence de certains risques liés au patient. Il est également recommandé d'utiliser un CSF à titre de soutien en vue d'éviter le recours aux réductions de doses ou les retards durant les traitements adjuvants, les traitements curatifs et les traitements ayant pour but de prolonger la survie. Les lignes directrices de l'Organisation européenne de recherche sur le traitement du cancer (OERTC) fournissent un algorithme d'évaluation des risques et de prise de décision sur l'utilisation prophylactique de G-CSF (Figure 1). De nombreux exemples de protocoles ayant un risque de NF $\geq 10 \%$ sont également énumérés dans les lignes directrices de l'OERTC.

Les infirmières en oncologie sont les principales championnes des soins de soutien pour les patients et jouent un rôle capital dans l'identification des patients présentant un risque élevé de NF; pourtant, les résultats de départ de l'AIM Higher Initiative, un programme d'amélioration de la qualité se déroulant actuellement, suggèrent que beaucoup d'infirmières n'évaluent pas systématiquement le risque de neutropénie chez leurs patients (Johnson, Moore \& Fortner, 2007). Cette initiative a pour but d'optimiser les soins de soutien en rehaussant l'évaluation des symptômes liés au cancer, l'éducation du patient et la gestion de cinq groupes de symptômes reliés à la chimiothérapie : l'anémie, la neutropénie, la diarrhée et la constipation, la nausée et les vomissements et enfin, la dépression et l'anxiété. Des 376 adultes atteints de cancer exam-

Au sujet des auteurs

Kelley Moore, inf., Lakeland, TN. Courriel : jkmg06@gmail.com

Barry Fortner, Ph.D., V.-P. principal, Scientific Affairs and Provider Services, P4 Healthcare, Lakeland, TN. Courriel : bfortner@p4healthcare.com 
inés dans 15 cliniques d'oncologie communautaires américaines dans le cadre de l'AIM Higher Initiative, ils n'étaient que 96 (26 \%) à avoir une quelconque preuve écrite d'une évaluation prétraitement du risque de neutropénie.

Il existe d'abondantes preuves en provenance d'études dirigées par des infirmières que le dépistage des patients à risque élevé de NF, suivi par une prophylaxie au G-CSF lorsque celle-ci est indiquée, pouvait rehausser les résultats pour le patient tels que réduire les retards dans l'administration des doses, minimiser les réductions de doses et éviter les hospitalisations liées à la NF (Lenhart, 2004; Donohue, 2006; Doyle, 2006; White, Maxwell, Michelson \& Bedell, 2005). Nous avons réalisé une étude afin de tester notre hypothèse à savoir qu'un processus normalisé d'évaluation du risque de NF aiderait les infirmières à jouer un rôle proactif dans la réduction du risque de neutropénie induite par la chimiothérapie. Les buts secondaires de l'étude étaient de documenter les perceptions des infirmières sur l'utilité d'un outil d'évaluation de ce risque et de dégager les interventions de soins aux patients étant les plus fréquemment mises en œuvre après l'évaluation.

\section{Méthodologie}

Cette étude exploratoire s'intitulait Feasibility of Utilizing the Amgen Chemotherapy-induced Neutropenia Risk Assessment Tool in Clinical Oncology [Faisabilité de l'utilisation, en oncologie clinique, de l'outil Amgen d'évaluation du risque de neutropénie induite par la chimiothérapie]. L'étude a été réalisée dans les 15 cliniques d'oncologie communautaires participant à l'AIM Higher Initiative, bien que l'étude soit un projet indépendant de cette initiative. Les infirmières ont été recrutées pour l'étude grâce au concours des chefs de file de la profession infirmière dans chacune des cliniques visées par l'Initiative. Entre juillet et décembre 2004, 15 infirmières œuvrant dans 15 sites de pratique indépendants se sont servies

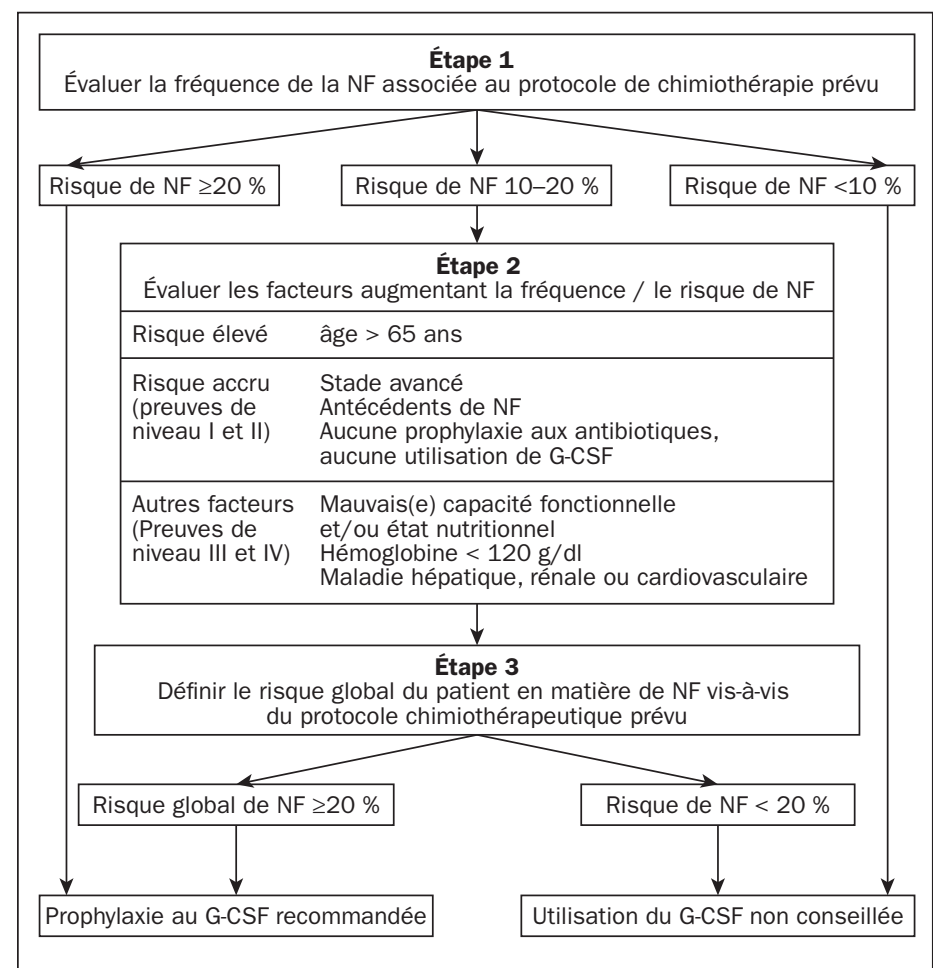

Figure 1. Algorithme de prise de décision de l'Organisation européenne de recherche sur le traitement du cancer relatif à l'utilisation prophylactique d'un facteur de stimulation des colonies granulocytaires (G-CSF). NF = neutropénie fébrile. Source : Aapro et al., 2006. Tiré du European Journal of Cancer, 42, 2433-2453, Copyright 2006, avec la permission d'Elsevier [traduction libre]. d'une liste de contrôle afin d'évaluer le risque de NF chez les 10 prochains patients adultes devant recevoir une chimiothérapie (soit un total de 150 patients) aiguillés vers elles à des fins d'évaluation initiale du patient et d'enseignement au patient; il n'était pas nécessaire que ces nouveaux patients soient vierges de tout traitement chimiothérapeutique mais ils devaient commencer un nouveau protocole de chimiothérapie. Nous n'exigions pas des patients qu'ils répondent à quelque autre critère d'admissibilité. Nous n'avons pas recherché leur consentement éclairé dans le cadre de l'étude puisque celle-ci visait à évaluer l'expérience des infirmières relativement à l'utilisation du nouvel outil d'évaluation des patients. Aucun renseignement personnel sur les patients n'a été fourni à l'équipe de recherche. En outre, l'outil d'évaluation demeurait dans le dossier de chaque patient, les chercheurs ne recevant que les évaluations de l'outil par les infirmières.

Nous avons utilisé une liste de contrôle fournie par Amgen Inc. (Figure 2); cet outil était complet et il incorporait les plus récentes données disponibles au moment du lancement de l'étude. Cet outil a également été employé dans d'autres études d'amélioration de la qualité comme celle entreprise par Doyle (2006). Cet outil particulier-une liste de contrôle-a été utilisé afin d'identifier les patients présentant un ou plusieurs facteurs de risque vis-à-vis de la NF et donc étant de bons candidats pour une thérapie prophylactique au facteur de croissance.

Après avoir effectué l'évaluation de risque, les infirmières ont consigné les facteurs de risque cernés et les interventions mises en œuvre

\section{Évaluation du patient pour la gestion de la neutropénie induite par la chimiothérapie}

\begin{tabular}{|c|c|}
\hline & patient \\
\hline & teur de risque lié à la chimiothérapie \\
\hline & $\begin{array}{l}\text { Protocole de chimiothérapie associé à un risque allant de } \\
\text { modéré à élevé de complications neutropéniques }\end{array}$ \\
\hline $\mathrm{Fac}$ & teurs de risque indépendants \\
\hline & Âge $>70$ ans \\
\hline & Neutropénie existante \\
\hline & Chimiothérapie intense préalable \\
\hline & Irradiation antérieure des os sources de moelle \\
\hline & Antécédents de NF récidivante lors de chimiothérapies antérieures \\
\hline & Mauvaise capacité fonctionnelle \\
\hline & Cancer de stade avancé \\
\hline & Fonction immunitaire affaiblie : p. ex. diabète, BPCO \\
\hline & Plaies ouvertes \\
\hline & Infection active de tissus \\
\hline & Sérum-albumine $\geq 3,5 \mathrm{~g} / \mathrm{dl}(\mathrm{LNH})$ \\
\hline & Taux élevé de LDH sérique (LNH) \\
\hline & Atteinte à la moelle osseuse \\
\hline & Patients subissant une thérapie à haute dose \\
\hline Th & rapie au facteur de croissance : besoins du patient \\
\hline & $\begin{array}{l}\text { Le patient est un candidat pour la thérapie au facteur de croissance } \\
\square \text { Fixer un rendez-vous pour l'injection. } \\
\square \text { Fournir une ordonnance de facteur de croissance. } \\
\square \text { Dispenser les cycles ultérieurs de la thérapie. }\end{array}$ \\
\hline & $\begin{array}{l}\text { Le patient n'est pas un bon candidat pour la thérapie au facteur } \\
\text { de croissance; surveiller étroitement son état. }\end{array}$ \\
\hline & ture du médecin \\
\hline
\end{tabular}

Figure 2. L'outil normalisé et fondé sur les preuves qui a été utilisé dans le cadre de l'étude afin d'évaluer les patients à risque élevé de neutropénie fébrile (NF). BPCO = Bronchopneumopathie chronique obstructive; LDH = lacticodéshydrogénase; LNH = lymphome non hodgkinien. [Traduction libre] 
à la lumière de ces résultats. Après chacune de ces interactions infirmière-patient, les infirmières remplissaient également un questionnaire développé par l'équipe de recherche au moyen duquel elles appréciaient le degré d'utilité de l'utilisation systématique de l'outil d'évaluation du risque par rapport aux soins du patient (Figure 3). Les questions avaient été élaborées dans le seul but d'aborder les objectifs de l'étude, à savoir (i) déterminer l'utilité de l'outil d'évaluation du risque de neutropénie induite par la chimiothérapie (NIC) dans le contexte de l'oncologie clinique; (ii) évaluer les perceptions des infirmières sur la manière dont l'outil peut faciliter la gestion proactive de la NIC; et enfin, (iii) mettre en évidence les plus courants des facteurs de risque liés aux patients ainsi que les mesures prises à la lumière des résultats de l'évaluation du risque. À chacune des questions correspondaient plusieurs réponses possibles. Les infirmières étaient priées d'exprimer dans quelle mesure elles étaient d'accord avec chaque réponse au moyen d'une échelle allant de 1 à 5 où 1 signifie Tout à fait en désaccord et 5 , Tout à fait d'accord.

Après que chacune des infirmières participantes avait utilisé l'outil d'évaluation du risque auprès de son dixième patient et rempli le questionnaire correspondant, elle remplissait également une enquête d'évaluation finale-elle aussi mise au point par l'équipe de recherche-laquelle servait à évaluer l'utilité globale de l'intégration de l'outil d'évaluation du risque dans la pratique clinique régulière (Figure 4). Nous demandions spécifiquement aux infirmières si elles incorporeraient dans leur pratique la liste de contrôle pour l'évaluation du risque de NIC utilisée dans le cadre de l'étude ou un outil similaire. Tout comme dans les autres questionnaires, les infirmières étaient invitées à exprimer leur accord avec l'énoncé au moyen d'une échelle allant de 1 à 5.
Nous avons fait appel à la statistique descriptive pour analyser les données et présenter les résultats en fonction de chaque site et de l'ensemble de l'échantillon. Des tables des fréquences ont été produites pour diverses variables nominales. Des indicateurs de variance aléatoire, notamment les écarts-types et les intervalles de confiance, ont été calculés pour certaines variables continues.

\section{Résultats}

Les15 infirmières répondantes de l'étude participaient déjà toutes à l'évaluation, à l'interrogation et à l'éducation de nouveaux patients (tableau 1). Elles avaient, en moyenne, 12 années d'expérience en oncologie. Sur les 150 patients concernés, 110 (73\%) étaient des femmes, et le cancer du sein et les cancers gynécologiques représentaient presque la moitié des sièges de cancer primaire.

Dans le questionnaire qu'elles remplissaient immédiatement après avoir mené chacune de leurs 10 évaluations du risque, les infirmières indiquaient que l'utilisation d'un outil normalisé les avait aidées à évaluer le risque de NF chez $72 \%$ des patients et à déterminer le degré de risque de NF chez 57 \% (Figure 5). La majorité des patients (83 patients, $55 \%$ ) était admissibles à un traitement prophylactique au G-CSF parce qu'ils devaient recevoir un protocole de chimiothérapie allant de modérément à fortement myélosuppresseur. De plus, une proportion substantielle des patients présentait des caractéristiques qui justifiaient l'administration d'une prophylaxie au G-CSF. Les facteurs de risque de NF les plus fréquemment cernés chez les patients était le stade avancé de leur cancer (46 patients, $31 \%$ ), leur âge $>70$ ans (33 patients, $22 \%$ ), des troubles de la fonction immunitaire (19 patients, $13 \%$ ) et une chimiothérapie antérieure de grande intensité (18 patients, 12 \%).

\section{Évaluation du risque de neutropénie}

Date : Responsable de l'entrevue

Instructions : Sélectionner la cote correspondant aux énoncés suivants dans le contexte de chaque utilisation de l'outil d'évaluation du risque :

1 = Tout à fait en désaccord $2=$ En désaccord $3=$ Neutre $4=D^{\prime}$ accord $5=$ Tout à fait d'accord

L'outil m'a aidée à déterminer le risque de neutropénie (1) (2) (3) (4) (5)

L'outil m'a aidée à déterminer le degré de gravité du risque de neutropénie

L'outil a aidé à déterminer s'il fallait utiliser une prophylaxie au G-CSF

L'outil a aidé à définir les questions majeures liées au remboursement de la thérapie au G-CSF

L'outil a permis de documenter le risque de neutropénie de manière claire et concise

Combien de temps le remplissage de l'outil d'évaluation du risque vous a-t-il pris?

L'outil d'évaluation du risque a-t-il abouti à un ou plusieurs des résultats ci-dessous :

Surveiller plus étroitement le patient pour ce qui est du risque de neutropénie

Utilisation prophylactique du G-CSF

Changement de la dose de chimiothérapie

Changement du calendrier d'administration de la chimiothérapie

Choix du protocole de chimiothérapie (1) (2) (3) (4) (5)

(1) (2) (3) (4) (5)

(1) (2) (3) (4) (5)

(1) (2) (3) (4) (5)

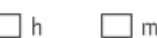

Oui ONon

Oui ONon

Oui ONon

O Oui ONon

Oui ONon

Commentaires

Figure 3. Questionnaire permettant d'évaluer le degré d'utilité pour les infirmières de l'utilisation systématique d'un outil d'évaluation du risque de NF. L'original est reproduit avec l'autorisation de Supportive Oncology Services. 
Pour la vaste majorité des patients (141 patients, $94 \%$ ), les résultats de l'évaluation du risque déclenchaient une ou plusieurs intervention(s) visant à réduire la durée de la neutropénie sévère induite par la chimiothérapie et de ses complications, notamment la NF et les infections. Chez 96 patients (64\%), l'intervention la plus souvent retenue était une surveillance plus étroite du patient concernant la NF. Les autres interventions étaient la prescription d'une prophylaxie au G-CSF (41 patients, $27 \%$ ), une modification $\mathrm{du}$ protocole de chimiothérapie (3 patients, $2 \%$ ) et enfin, une modification de la dose de chimiothérapie (1 patient, 0,7\%). La cinquième option figurant dans le questionnaire, changer le calendrier de la chimiothérapie, n’a été sélectionnée par aucune infirmière.

Lors de l'évaluation finale, presque toutes les infirmières, soit 13 sur 15 (87\%), ont déclaré qu'il leur avait été facile d'utiliser un outil d'évaluation du risque. En outre, 67 \% ont rapporté que l'utilisation de l'outil les avait aidées à déterminer quels patients étaient à risque élevé de NF et $73 \%$ ont signalé que leur établissement projetait d'évaluer systématiquement le risque de NF à l'avenir au moyen du même outil ou d'une méthode similaire. Les deux infirmières qui se sont dites peu susceptibles d'utiliser à l'avenir cet outil ou un outil semblable faisaient valoir que la liste de contrôle ne permettait d'évaluer qu'un seul symptôme chez les patients, à savoir la neutropénie. Étant donné que les infirmières évaluent systématiquement de multiples symptômes et effets indésirables chez les patients, elles avançaient qu'un outil qui leur permettrait d'évaluer de multiples symptômes leur serait d'une plus grande utilité.

\begin{tabular}{|c|c|c|c|c|c|}
\hline & \multicolumn{2}{|c|}{$\square \square \square$} & \multicolumn{3}{|c|}{$\underset{\text { Suljet vo }}{\square} \square$} \\
\hline \multicolumn{6}{|c|}{ Improving Assessment, Information and Management of Chemotherapy Toxicities } \\
\hline \multicolumn{6}{|l|}{$\begin{array}{l}\text { Évaluation finale de l'outil } \\
\text { d'évaluation du risque de neutropénie }\end{array}$} \\
\hline Date de l'enquête : $\square \square$ / $\square \square / \square \square$ Responsable de l'entr & trevue & & & & \\
\hline \multicolumn{6}{|c|}{$\begin{array}{l}\text { Instructions : Sélectionner la cote correspondant aux énoncés suivants dans le contexte de } \\
\text { toutes les utilisations de l'outil d'évaluation du risque de neutropénie : } \\
1=\text { Tout à fait en désaccord } 2=\text { En désaccord } 3=\text { Neutre } 4=\text { D'accord } 5=\text { Tout à fait d'accord }\end{array}$} \\
\hline L’outil était facile à utiliser. & (1) & (2) & (3) & (4) & (5) \\
\hline $\begin{array}{l}\text { L'outil a aidé à identifier les patients susceptibles } \\
\text { d'être atteints de neutropénie. }\end{array}$ & (1) & (2) & (3) & (1) & (5) \\
\hline $\begin{array}{l}\text { L'outil a encouragé l'évaluation de facteurs qui ne sont } \\
\text { pas systématiquement abordés auprès des patients. }\end{array}$ & (1) & (2) & (3) & (4) & (5) \\
\hline Nous prévoyons utiliser l'outil dans le cadre de la pratique régulière. & (1) & (2) & (3) & (4) & (5) \\
\hline $\begin{array}{l}\text { Nous prévoyons adopter l'outil dans un format qui } \\
\text { pourra être utilisé dans le cadre de la pratique régulière. }\end{array}$ & (1) & (2) & (3) & (4) & (5) \\
\hline $\begin{array}{l}\text { Nous prévoyons commencer à évaluer systématiquement } \\
\text { le risque de neutropénie par le biais d'une méthode } \\
\text { autre que l'outil d'évaluation du risque. }\end{array}$ & (1) & (2) & (3) & (4) & \\
\hline Comment pourrait-on améliorer l'utilité de l'outil? & & & & & \\
\hline
\end{tabular}

Quelles autres suggestions avez-vous?

Veuillez décrire les obstacles à l'évaluation du risque dans votre clinique.

Figure 4. Questionnaire final évaluant l'utilité globale de l'intégration d'un outil d'évaluation du risque dans la pratique clinique régulière. Original reproduit avec la permission de Supportive Oncology Services.

\section{Discussion et conclusions}

Les résultats de cette étude menée par une infirmière indiquent qu'il est possible et très utile pour les infirmières en oncologie d'évaluer le risque de NF chez les patients adultes avant même le démarrage de la chimiothérapie. Les infirmières ont ainsi décelé des facteurs de risque chez quasiment tous les 150 patients qu'elles ont évalués (94\%), ce qui les a amenées à prendre des mesures en vue de réduire l'incidence de la neutropénie sévère induite par la chimiothérapie et ses complications.

Un outil normalisé d'évaluation du risque tel que celui que nous avons utilisé dans cette étude est applicable à tout un éventail de

\begin{tabular}{|c|c|}
\hline \multicolumn{2}{|l|}{ Infirmières $(\mathrm{N}=15)$} \\
\hline Âge, années, moyenne (é.-t.) & $39,0(10,8)$ \\
\hline $\begin{array}{l}\text { Expérience en oncologie, années, } \\
\text { moyenne (é.-t.) }\end{array}$ & $12,0(9,0)$ \\
\hline $\begin{array}{l}\text { Participent aux entrevues avec les nouveaux } \\
\text { patients et à leur évaluation, \% }\end{array}$ & 100 \\
\hline $\begin{array}{l}\text { Participent à l'éducation des nouveaux } \\
\text { patients, \% }\end{array}$ & 100 \\
\hline \multicolumn{2}{|l|}{ Patients $(\mathrm{N}=150)$} \\
\hline Âge, années, moyenne (é.-t.) & $58,3(12,7)$ \\
\hline De sexe féminin, nombre (\%) & $110(73,3)$ \\
\hline \multicolumn{2}{|l|}{ Siège du cancer primaire, nombre (\%) } \\
\hline Sein & $50(33,3)$ \\
\hline Sphère gynécologique & $24(16,0)$ \\
\hline Poumon & $21(14,0)$ \\
\hline Sphére gastrointestinale & $18(12,0)$ \\
\hline Lymphome non hodgkinien & $11(7,3)$ \\
\hline Autre & $26(17,4)$ \\
\hline \multicolumn{2}{|l|}{ Stade du cancer primaire, nombre (\%) } \\
\hline I & $22(14,7)$ \\
\hline II & $41(27,3)$ \\
\hline III & $35(23,3)$ \\
\hline IV & $30(20,0)$ \\
\hline Données manquantes & $22(14,7)$ \\
\hline
\end{tabular}

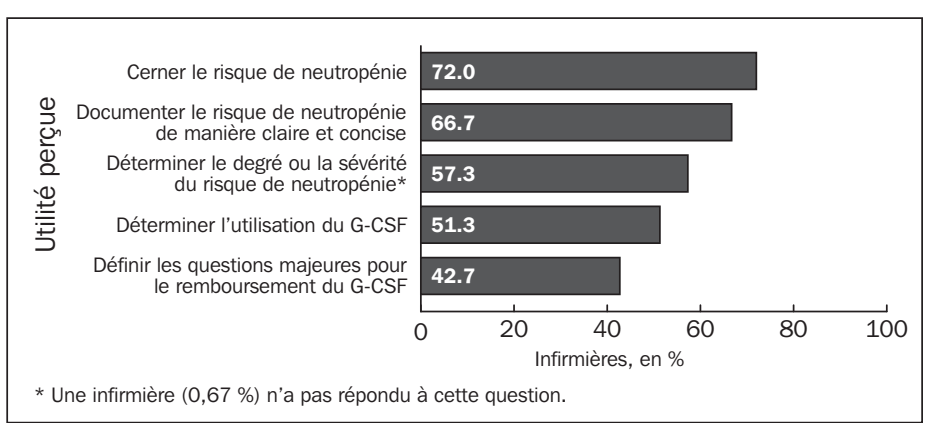

Figure 5. Perceptions des infirmières relativement à l'utilité d'un outil d'évaluation du risque de neutropénie fébrile. G-CSF = Facteur de stimulation des colonies granulocytaires. 
types de tumeurs. Il s'intègre aisément dans la pratique régulière, si l'on se fie aux $87 \%$ des infirmières participant à l'étude qui l'ont défini comme étant d'une utilisation aisée. L'information sur les risques ne figure pas toujours dans le dossier du patient avant le traitement, et une telle liste de contrôle fournit à l'infirmière le cadre dont elle a besoin pour poser des questions supplémentaires lors de l'entrevue avec le patient. L'emploi d'un outil normalisé devrait aussi faciliter le maintien d'une qualité uniforme concernant l'évaluation des patients à l'échelle de l'établissement.

L'opinion générale ressortant des lignes directrices et des recommandations de diverses organisations des États-Unis, d'Europe et du Canada est que les évaluations des risques devraient être réalisées avant que ne débute le premier cycle de chimiothérapie (Aapro et al., 2006; Kouroukis et al., 2008; NCCN, 2008; Smith et al., 2006). Toutefois, chacune des trois lignes directrices propose une orientation légèrement différente sur les facteurs de risque qu'il convient d'évaluer pour la NF induite par la chimiothérapie. Les infirmières en oncologie doivent concentrer leur attention sur l'adaptation de ces lignes directrices nationales et internationales fondées sur les preuves en vue d'élaborer des outils d'évaluation des risques qui soient pertinents pour leur propre contexte de pratique et leurs propres clientèles (Maxwell \& Stein, 2006). Par exemple, Donohue a cerné les facteurs de risque relatifs à la neutropénie en se basant sur les écrits et les lignes directrices publiés et en a tiré une liste simplifiée de facteurs s'appliquant à l'ensemble des patients atteints de cancers autres que les leucémies (Donohue, 2006). Quel que soit l'outil particulier retenu, il convient de l'adjoindre au dossier du patient afin d'en partager l'information avec les autres soignants.

À mesure que de nouvelles preuves émergent et que le raffinement des outils d'évaluation des risques se poursuit, il sera possible d'identifier plus précisément les patients exigeant une prophylaxie au G-CSF et/ou ayant besoin d'interventions infirmières additionnelles afin de prévenir l'apparition de la NF ou de la gérer (Lyman, Lyman \& Agboola, 2005). De nouvelles connaissances sur les facteurs de risque ont même vu le jour durant le déroulement de notre étude et ont abouti à des changements aux lignes directrices qui faisaient consensus. Par exemple, au moment du démarrage de notre étude, un risque de NF était associé aux patients de plus de 70 ans, selon les lignes du NCCN. Depuis, ces lignes directrices font état d'un risque de NF plus élevé chez les patients de 65 ans et plus (Aapro et al., 2006; Smith et al., 2006; NCCN, 2008). L'on risque moins d'omettre des facteurs de risque nouvellement dégagés si l'on utilise un outil normalisé faisant l'objet de fréquentes révisions afin qu'il reflète les conclusions les plus récentes.

Plusieurs études dirigées par des infirmières (Donohue, 2006; Doyle, 2006; Lenhart, 2004; White et al., 2005) ont montré que l'identification systématique des patients à risque élevé de NF, suivie par une prophylaxie au G-CSF le cas échéant, améliore les résultats pour le patient en ce qui concerne les manifestations de la neutropénie. Parmi les améliorations, on peut citer une réduction importante de la nécessité de retarder les doses de la chimio- thérapie (Donohue, 2006), des réductions significatives du taux d'hospitalisation liée à la NF ainsi que la durée des séjours à l'hôpital (Doyle, 2006). La présente étude prend appui sur les travaux antérieurs pour confirmer que l'évaluation systématique préalable à l'administration de la chimiothérapie amène les infirmières et d'autres professionnels de la santé à prendre des mesures en vue de minimiser le risque de NF chez leurs patients.

Les infirmières rencontrent souvent des obstacles dans la mise en œuvre d'un nouvel outil, notamment le manque de temps et la réticence des collègues de travail. Il importe d'adopter un outil facile à utiliser, basé sur un questionnaire existant ou sur un algorithme élaboré à partir de preuves (voir la Figure 1), d'expliquer aux médecins de la clinique et à d'autres membres de son personnel que l'emploi de l'outil se traduira par une amélioration des soins et un rehaussement de l'efficacité. Maxwell et Stein (2006) présentent des suggestions détaillées à l'intention des infirmières désirant intégrer dans leur pratique clinique les lignes directrices relatives à l'évaluation de la NF, dont un modèle de liste de contrôle, un modèle d'algorithme aidant à déterminer quels patients ont besoin d'une prophylaxie au CSF et une liste de stratégies permettant de surmonter les obstacles.

Il est également important de solliciter la rétroaction des membres de l'équipe afin d'aborder les besoins des autres membres du personnel. Par exemple, quelques-unes des infirmières participant à notre étude ont suggéré qu'un outil permettant d'évaluer de multiples symptômes chez les patients serait plus utile qu'un outil se limitant à l'évaluation d'un seul effet indésirable potentiel, à savoir la neutropénie. Les chefs de file en soins infirmiers de l'AIM Higher Initiative ont ainsi mis au point un outil d'évaluation des risques relatifs à de multiples symptômes (Johnson et al., 2007). L'intégration de l'évaluation des risques liés à bon nombre de symptômes dans les dossiers médicaux électroniques est une autre stratégie qui pourrait aider à surmonter cet obstacle.

Les infirmières sont les membres des équipes multidisciplinaires de soins aux patients qui occupent une place privilégiée pour évaluer, chez ces derniers, le risque de NF. En utilisant un outil à la fois simple et pratique, les infirmières ont la possibilité d'exercer une profonde incidence positive sur les troubles associés à la neutropénie chez les patients subissant un traitement de chimiothérapie myélosuppresseur.

\section{Remerciements}

Les auteurs souhaitent reconnaître l'aide d'Amgen Canada qui a fourni un soutien financier à la rédaction d'écrits médicaux par de tierces parties. Les auteurs reconnaissent également les apports, sous forme d'écrits médicaux, de Supriya Srinivasan, Ph.D., et de Faith Reidenbach, de la société Scientia Medical Communications.

L'étude a été conçue et réalisée par Supportive Oncology Services, Inc et financée par Amgen, Inc. L'outil d'évaluation du risque de neutropénie utilisé dans le cadre de l'étude a été fourni en 2004 par Amgen, Inc.

\section{Références}

Aapro, M.S., Cameron, D.A., Pettengell, R., Bohlius, J., Crawford, J., Ellis, M., et al. (2006). EORTC guidelines for the use of granulocyte-colony stimulating factor to reduce the incidence of chemotherapyinduced febrile neutropenia in adult patients with lymphomas and solid tumours. European Journal of Cancer, 42(15), 2433-2453.

Bonadonna, G., Valagussa, P., Moliterni, A., Zambetti, M., \& Brambilla, C. (1995). Adjuvant cyclophosphamide, methotrexate, and fluorouracil in node-positive breast cancer: The results of 20 years of follow-up. New England Journal of Medicine, 332(14), 901-906.
Budman, D.R., Berry, D.A., Cirrincione, C.T., Henderson, I.C., Wood, W.C., Weiss, R.B., et al. (1998). Dose and dose intensity as determinants of outcome in the adjuvant treatment of breast cancer. The Cancer and Leukemia Group B. Journal of the National Cancer Institute, 90(16), 1205-1211.

Caggiano, V., Weiss, R.V., Rickert, T.S., \& Linde-Zwirble, W.T. (2005). Incidence, cost, and mortality of neutropenia hospitalization associated with chemotherapy. Cancer, 103(9), 1916-1924. 
Cordonnier, C., Herbrecht, R., Buzyn, A., Leverger, G., Leclercq, R., Nitenberg, G., et al. (2005). Risk factors for Gram-negative bacterial infections in febrile neutropenia. Haematologica, 90(8), 1102-1109.

Dale, D.C. (2002). Colony-stimulating factors for the management of neutropenia in cancer patients. Drugs, 62(supp 1), 1-15.

Desjardins, B., \& Meilleur, M.-C. (2005). Epidemiology of febrile neutropenia in Canadian provinces: Hospitalization-based incidence. Presented at the 7th International Symposium on Febrile Neutropenia, January 27-29, 2005, Seville, Spain.

Donohue, R. (2006). Development and implementation of a risk assessment tool for chemotherapy-induced neutropenia. Oncology Nursing Forum, 33(2), 347-352.

Doyle, A. (2006). Prechemotherapy assessment of neutropenic risk. Oncology Nurse Edition, 20(10, Suppl. 7). Retrieved June 6, 2007, from http://www.cancernetwork/nurses-edition

Epelbaum, R., Faraggi, D., Ben-Arie, Y., Ben-Shahar, M., Haim, N., Ron, Y., et al. (1990). Survival of diffuse large cell lymphoma. A multivariate analysis including dose intensity variables. Cancer, 66(6), 1124-1129.

Gaytán-Martínez, J., Mateos-Garcia, E., Sánchez-Cortes, E., GonzálezLlaven, J., Casanova-Cardiel, L.J., Fuentes-Allen, J.L. (2000). Microbiological findings in febrile neutropenia. Archives of Medical Research, 31(4), 388-392.

Johnson, G.D., Moore, K., \& Fortner, B. (2007). Baseline evaluation of the AIM Higher Initiative: Establishing the mark from which to measure. Oncology Nursing Forum, 34(3), 729-734.

Kouroukis, C.T., Chia, S., Verma, S., Robson, D., Desbiens, C., Cripps, C., et al. (2008). Canadian supportive care recommendations for the management of neutropenia in patients with cancer. Curr Oncol, 15(1), 9-23.

Kuderer, N.M., Dale, D.C., Crawford, J., Cosler, L.E., \& Lyman, G.H. (2006). Mortality, morbidity, and cost associated with febrile neutropenia in adult cancer patients. Cancer, 106(10), 2258-2266.

Kwak, L.W., Halpern, J., Olshen, R.A., \& Horning, S.J. (1990). Prognostic significance of actual dose intensity in diffuse largecell lymphoma: Results of a tree-structured survival analysis. Journal of Clinical Oncology, 8(6), 963-977.
Lenhart, C. (2004). Performance improvement to ensure chemotherapy dose delivery. Oncology Supportive Care Quarterly, 2(4), 6-14.

Lyman, G.H., Dale, D.C., \& Crawford, J. (2003). Incidence and predictors of low dose-intensity in adjuvant breast cancer chemotherapy: A nationwide study of community practices. Journal of Clinical Oncology, 21(24), 4524-4531.

Lyman, G.H., Dale, D.C., Friedberg, J., Crawford, J., \& Fisher, R.I. (2004). Incidence and predictors of low chemotherapy doseintensity in aggressive non-Hodgkin's lymphoma: A nationwide study. Journal of Clinical Oncology, 22(21), 4302-4311.

Lyman, G.H. (2005). Guidelines of the National Comprehensive Cancer Network on the use of myeloid growth factors with cancer chemotherapy: A review of the evidence. Journal of the National Comprehensive Cancer Network, 3(4), 557-571.

Lyman, G.H., Lyman, C.H., \& Agboola, O. (2005). Risk models for predicting chemotherapy-induced neutropenia. Oncologist, 10(6), 427-437.

Maxwell, C., \& Stein, A. (2006). Implementing evidence-based guidelines for preventing chemotherapy-induced neutropenia: From paper to clinical practice. Community Oncology, 3, 530-536.

National Comprehensive Cancer Network. Myeloid Growth Factors: The National Comprehensive Cancer Network Clinical Practice Guidelines in Oncology, version 1.2008. January 2, 2008. Retrieved August 4, 2008, from http://www.nccn.org

Smith, T.J.C., Khatcheressian, J., Lyman, G.H., Ozer, H., Armitage, J.O., Balducci, L., et al. (2006). 2006 update of recommendations for the use of white blood cell growth factors: An evidence-based clinical practice guideline. Journal of Clinical Oncology, 24(19), 3187-3205.

Vogel, C.L., Wojtukiewicz, M.Z., Carroll, R.R., Tjulandin, S.A., BarajasFigueroa, L.J., Wiens, B.L., et al. (2005). First and subsequent cycle use of pegfilgrastim prevents febrile neutropenia in patients with breast cancer: A multicenter, double-blind, placebo-controlled phase III study. Journal of Clinical Oncology, 23(6), 1178-1184.

White, N., Maxwell, C., Michelson, J., \& Bedell, C. (2005). Protocols for managing chemotherapy-induced neutropenia in clinical oncology practices. Cancer Nursing, 28(1), 62-69. 